\title{
SPECTROSCOPY OF THE LARGEST EVER $\gamma$-RAY-SELECTED BL LAC SAMPLE
}

\author{
Michael S. Shaw ${ }^{1}$, Roger W. Romani ${ }^{1}$, Garret Cotter ${ }^{2}$, Stephen E. Healey ${ }^{1}$, Peter F. Michelson ${ }^{1}$, Anthony C. \\ S. Readhead ${ }^{3}$, Joseph L. Richards ${ }^{3}$, Walter MaX-Moerbeck ${ }^{3}$, Oliver G. King ${ }^{3}$, And William J. Potter ${ }^{2}$ \\ ${ }^{1}$ Department of Physics/KIPAC, Stanford University, Stanford, CA 94305, USA \\ 2 Department of Astrophysics, University of Oxford, Oxford OX1 3RH, UK \\ ${ }^{3}$ Department of Astronomy, California Institute of Technology, Pasadena, CA 91125, USA \\ Received 2012 October 29; accepted 2012 December 28; published 2013 January 31
}

\begin{abstract}
We report on spectroscopic observations covering most of the 475 BL Lacs in the second Fermi Large Area Telescope (LAT) catalog of active galactic nuclei (AGNs). Including archival measurements (correcting several erroneous literature values) we now have spectroscopic redshifts for 44\% of the BL Lacs. We establish firm lower redshift limits via intervening absorption systems and statistical lower limits via searches for host galaxies for an additional $51 \%$ of the sample leaving only $5 \%$ of the BL Lacs unconstrained. The new redshifts raise the median spectroscopic $\tilde{z}$ from 0.23 to 0.33 and include redshifts as large as $z=2.471$. Spectroscopic redshift minima from intervening absorbers have $\tilde{z}=0.70$, showing a substantial fraction at large $z$ and arguing against strong negative evolution. We find that detected BL Lac hosts are bright ellipticals with black hole masses $M_{\bullet} \sim 10^{8.5}-10^{9}$, substantially larger than the mean of optical AGNs and LAT Flat Spectrum Radio Quasar samples. A slow increase in $M_{\text {. with }} z$ may be due to selection bias. We find that the power-law dominance of the optical spectrum extends to extreme values, but this does not strongly correlate with the $\gamma$-ray properties, suggesting that strong beaming is the primary cause of the range in continuum dominance.
\end{abstract}

Key words: BL Lacertae objects: general - galaxies: active - gamma rays: galaxies - quasars: general - surveys

Online-only material: color figures, figure set, machine-readable table

\section{INTRODUCTION}

The Fermi Second Source Catalog (2FGL; Nolan et al. 2012) lists the 1873 most significant sources detected by the Large Area Telescope (LAT; Atwood et al. 2009) during Fermi's first two years of sky survey observations. The majority of these sources are associated with jet-dominated active galactic nuclei (AGNs), the so-called blazars, many of which are bright, compact radio sources. There are, in fact, 1121 such associations (1017 at $|b|>10^{\circ}$ ), collected in the Second Catalog of AGNs Detected by the Fermi LAT (2LAC; Ackermann et al. 2011). These AGNs are further classified as Flat Spectrum Radio Quasars (FSRQs) where the optical spectrum is dominated by thermal disk and broad-line region emission, BL Lacs (BLLs), where the optical spectrum is dominated by continuum synchrotron radiation, and a collection of miscellaneous, mostly low-luminosity related sources. In $2 \mathrm{LAC}$, the sample included 410 BLLs, 357 FSRQs, 28 AGNs of other type (generally low $z$, lower luminosity Seyferts), and 326 AGNs of (then) unknown type.

These "blazars" (BLL and FSRQ) are the brightest extraGalactic point sources in the microwave and $\gamma$-ray bands; studies of their population and evolution are central topics in high-energy astrophysics. To support such studies we have acquired sensitive spectroscopic observations of this sample. In a companion paper (Shaw et al. 2012, hereafter S12), we reported on measurements of a large fraction of the FSRQs. Here we concentrate on the BL Lac objects. Our study has also found types for some of the unclassified blazars; the "unknowns" have now decreased to $215(19 \%)$, and the confirmed BLLs have increased to 475 ( $42 \%$ of all $2 \mathrm{LAC}$ AGNs).

In Section 2, we outline the sample properties, data collection, and data reduction steps. We also summarize principal features of the spectra. In Section 3, we describe our spectroscopic constraints on the redshift, including a technique to provide uniform redshift limits based on searches for host galaxy emission. In Section 4, we give estimates of the BLL black hole masses. We turn to comments on the principal BLL feature, the non-thermal dominance in the optical, in Section 5, and conclude with general remarks in Section 6.

In this paper, we assume an approximate concordance cosmology: $\Omega_{m}=0.3, \Omega_{\Lambda}=0.7$, and $H_{0}=70 \mathrm{~km} \mathrm{~s}^{-1} \mathrm{Mpc}^{-1}$.

\section{OBSERVATIONS AND DATA REDUCTION}

\subsection{The BLL Sample}

BLLs were originally identified as optical violently variable AGNs, and are often characterized by an optical continuum dominated by synchrotron emission. Their broadband spectral energy distribution (SED) is described by a synchrotron component peaking in the far-infrared to X-ray bands and an Inverse Compton (IC) component peaking in the $\mathrm{MeV}$ to $\mathrm{TeV}$ range. In the radio these sources display strong core dominance. According to the unified model (Urry \& Padovani 1995), BLLs are the beamed counterparts of the FR I radio galaxy population, while the FSRQs are associated with FR IIs. However, the principal BLL characteristic, a dominant and varying synchrotron/ IC continuum, is a sign of a powerful jet whose emission is beamed closely toward the Earth line of sight. Thus, the distinction between the traditional BLL and the FSRQ is sensitive to the precise state and orientation of the jet (e.g., Giommi et al. 2012) and, indeed, variations in jet power or direction can bring individual sources in or out of the BLL class (S12).

Our own assignment of the BLL label follows a pragmatic "optical spectroscopic" definition (Marcha et al. 1996): these are blazars with no emission lines greater than $5 \AA$ observed equivalent width, and a limit on any possible $4000 \AA$ spectral break of $<40 \%$ (Marcha et al. 1996; Healey et al. 2008; Shaw 
Table 1

Sample Completeness

\begin{tabular}{lcccc}
\hline \hline Set & Total & Spec $z$ & $z_{\min }$ & Unk. $z$ \\
\hline 2LAC & 1121 & & & \\
BLL & 475 & 209 & 241 & 25 \\
LBL & $72(21 \%)$ & $35(20 \%)$ & $36(23 \%)$ & \\
IBL & $91(26 \%)$ & $41(24 \%)$ & $49(31 \%)$ & \\
HBL & $187(53 \%)$ & $98(56 \%)$ & $73(46 \%)$ & \\
\hline
\end{tabular}

Notes. BLL includes 65 AGNs so classified since the 2LAC paper. Three hundred forty-nine BLLs have 2LAC SED subclasses; percentages give the breakdown. One hundred seventy-four of the spectroscopic redshifts and 158 of the lower limits have subclasses; percentage breakdowns are given.

et al. 2009). For transition objects with varying continuum emission, we retain the BLL label if it has ever been confirmed to be a BLL with a high-quality spectrum, even if subsequently observed in an FSRQ state. Within the BLL population it is common to classify sources based on the peak frequency of their SED's synchrotron component, as estimated from radio/ optical/X-ray flux ratios, separating the sources into lowpeak $\left(v_{\text {peak }}<10^{14} \mathrm{~Hz}\right.$, LBL $)$, intermediate-peak $\left(10^{14} \mathrm{~Hz}<\right.$ $\left.v_{\text {peak }}<10^{15} \mathrm{~Hz}, \mathrm{IBL}\right)$, and high-peak ( $\left.v_{\text {peak }}>10^{15} \mathrm{~Hz}, \mathrm{HBL}\right)$ sources. We adopt here the LBL/IBL/HBL designations from 2LAC, which provides such subclasses for $74 \%$ of all $2 \mathrm{LAC}$ BLLs, and $83 \%$ of those with spectroscopic redshifts (Table 1).

The evolution of BLL has long been controversial, and it has been claimed that they are predominantly a low redshift population, showing strong "negative" evolution (e.g., Beckmann et al. 2003), especially for the HBL class (Rector et al. 2000). One challenge to studies of the cosmological evolution of these sources is the difficulty of obtaining redshifts from their nearly featureless, continuum-dominated spectra. Indeed, many of the early studies using X-ray or radio-selected samples had highly incomplete redshift measurements, even though the samples were confined to relatively bright sources. Uncertainty in extrapolating from the measured set of redshifts complicated population interpretations. In the Fermi era, this issue becomes critical, as the large BLL contribution to the LAT source population and the hard BLL $\gamma$-ray spectra ensure that these sources are a major fraction of the cosmic $\gamma$-ray background and may, indeed dominate the LAT background at high energies (M. Ajello et al., in preparation).

Since the LAT provides a large, uniform, sensitivity-limited ( flux-limited) blazar sample, it provides a new opportunity to make progress on these questions. Important to interpreting the LAT blazars are the strong correlations between the LAT-detected (IC) part of the SED and the synchrotron component covering the optical band. The synchrotron peak location determines the subclassification, but also correlates with the intensity and LAT-band spectral index of the IC component, which in turn affects the depth of the LAT sample. Further, as illustrated in this paper, the synchrotron peak and intensity also affect the difficulty of optical spectroscopic measurements. Since we wish to recover the detailed evolution of the blazars, preferably also following differences among the LBL/IBL/HBL subclasses (or even better, a physical parent property leading to these subclasses), one needs a detailed treatment of both the $\gamma$-ray (e.g., Ajello et al. 2012) and optical selection effects. We reserve such analysis for a future study, noting here only the most prominent trends in the measured optical sample. Of course, characterization and minimization of the optical biases are greatly aided by high redshift completeness, the goal of the present paper.
We have accordingly studied the 2LAC sample over a number of years with a wide variety of telescopes, striving to be as complete as possible. We here report on observations of 278 BLL objects and 19 other Fermi blazars, not included in S12. We further analyzed 75 Sloan Digital Sky Survey (SDSS) spectra, treating them in the same manner as our new observations. Note that unless we suspected that a published redshift was erroneous, we generally did not obtain new spectra of many of the brighter, famous BLL with redshifts in the literature. In several cases when we obtained new data it strongly contradicted the literature redshift, either from a new secure spectroscopic $z$ or from an intervening absorber at larger $z$. In the end we retained 107 redshifts from literature values; however, for several early BLL $z \mathrm{~s}$ we have only inspected plotted spectra (of varying qualities); we suspect that at least a few erroneous values remain in this set. Our new data provide new spectroscopic redshifts for 102 objects and secure lower limits for many more, as summarized in Table 1. This brings the 2LAC BLL sample to $44 \%$ redshift completeness, and $95 \%$ completeness including redshift limits.

\subsection{Observations}

The quest for high completeness has driven us to employ medium and large telescopes in both hemispheres. Observations were obtained with the Marcario Low Resolution Spectrograph (LRS) on the Hobby-Eberly Telescope (HET), with the ESO Faint Object Spectrograph and Camera (EFOSC2; Buzzoni et al. 1984) and ESO Multi-Mode Instrument (EMMI; Dekker et al. 1986) on the New Technology Telescope at La Silla Observatory (NTT), with the Goodman High Throughput Spectrograph (GHTS) on the Southern Astrophysical Research (SOAR) Telescope, with the Double Spectrograph (DBSP) on the 200" Hale Telescope at Mt. Palomar, with the Focal Reducer and low dispersion Spectrograph (FORS2; Appenzeller et al. 1998) on the Very Large Telescope at Paranal Observatory (VLT), and with the Low Resolution Imaging Spectrograph (LRIS) at the W. M. Keck Observatory (WMKO). Observational configurations and objects observed are listed in Table 2.

Since many of these sources were not classified at the time of observation, we often initially obtained only sufficient signal-tonoise ratio $(\mathrm{S} / \mathrm{N})$ to identify the redshift of an FSRQ, or to firmly establish the source as a BLL. Also, with the variety of telescope configurations and varying observing conditions, the quality of the spectra are not uniform: resolutions vary from 4 to $17 \AA$, exposure times from $180 \mathrm{~s}$ to $2400 \mathrm{~s}$, and telescope diameters from $3.58 \mathrm{~m}$ to $10 \mathrm{~m}$. S/N per resolution element varies from 10 to $>300$. In a number of cases follow-on observations with higher $\mathrm{S} / \mathrm{N}$ and/or higher spectral resolution allowed us to more carefully study confirmed BLL lacking redshifts. Here we discuss the most constraining spectrum or spectrum average for each source, referring to this as the "primary" spectrum.

All spectra are taken at the parallactic angle, except for LRIS spectra using the atmospheric dispersion corrector, where we observed in a north-south configuration. In a few cases, we rotated the slit angle to minimize contamination from a nearby star. At least two exposures are taken of every target for cosmic ray cleaning. Typical exposure times are $2 \times 900 \mathrm{~s}$.

\subsection{Data Reduction Pipeline}

Data reduction was performed with the IRAF package (Tody 1986; Valdes 1986) using standard techniques. Data was overscan (where applicable) and bias subtracted. Dome flats were 
Table 2

Observing Configurations

\begin{tabular}{|c|c|c|c|c|c|c|c|}
\hline Telescope & Instrument & $\begin{array}{c}\text { Resolution } \\
(\AA)\end{array}$ & $\begin{array}{l}\text { Slit Width } \\
\quad\left({ }^{\prime \prime}\right)\end{array}$ & Objects & Filter & $\begin{array}{c}\lambda_{\min } \\
(\AA)\end{array}$ & $\begin{array}{c}\lambda_{\max } \\
(\AA)\end{array}$ \\
\hline HET & LRS & 15 & 2 & 41 & GG385 & 4150 & 10500 \\
\hline HET & LRS & 8 & 1 & 8 & GG385 & 4150 & 10500 \\
\hline NTT & EFOSC2 & 16 & 1 & 31 & $\ldots$ & 3400 & 7400 \\
\hline NTT & EMMI & 12 & 1 & 1 & $\cdots$ & 4000 & 9300 \\
\hline Palomar $200^{\prime \prime}$ & DBSP & $5 / 15$ & 1 & 4 & $\ldots$ & 3100 & 8100 \\
\hline Palomar $200^{\prime \prime}$ & DBSP & $5 / 15$ & 1.5 & 5 & $\cdots$ & 3100 & 8100 \\
\hline Palomar $200^{\prime \prime}$ & DBSP & $5 / 9$ & 1.5 & 42 & $\cdots$ & 3100 & 8100 \\
\hline SOAR & GHTS & 6 & 0.84 & 2 & $\cdots$ & 3200 & 7200 \\
\hline VLT & FORS2 & 11 & 1 & 14 & $\cdots$ & 3400 & 9600 \\
\hline VLT & FORS2 & 17 & 1.6 & 16 & $\cdots$ & 3400 & 9600 \\
\hline WMKO & LRIS & $4 / 7$ & 1 & 90 & $\cdots$ & 3100 & 10500 \\
\hline WMKO & LRIS & $4 / 9$ & 1 & 40 & $\cdots$ & 3100 & 10500 \\
\hline
\end{tabular}

Note. For DBSP and LRIS the blue and red channels are split by a dichroic at $5600 \AA$ A; the listed resolutions are for blue and red sides, respectively.

taken at the beginning of every night, the spectral response was removed, and all data frames were flat-fielded. Wavelength calibration employed arc lamp spectra and was confirmed with checks of night skylines. We employed an optimal extraction algorithm (Valdes 1992) to maximize the final S/N. For HET spectra, care was taken to use sky windows very near the longslit target position so as to minimize spectroscopic residuals caused by fringing in the red, whose removal is precluded by the rapidly varying HET pupil. Spectra were visually cleaned of residual cosmic ray contamination affecting only individual exposures.

We performed spectrophotometric calibration using standard stars from Oke (1990) and Bohlin (2007). In most cases, standard exposures were available from the data night. On the queue-scheduled HET, and during our queue-scheduled VLT observations, standards from subsequent nights were sometimes used. At all other telescopes, multiple standard stars were observed per night under varying atmospheric conditions and different air masses. The sensitivity function was interpolated between standard star observations when the solution was found to vary significantly with time.

For blue objects, broad-coverage spectrographs can suffer significant second-order contamination. In particular, the standard HET configuration using a Schott GG385 long-pass filter permitted second-order effects redward of $7700 \AA$. The effect on object spectra was small, but for blue WD spectrophotometric standards, second-order corrections were needed for accurate determination of the sensitivity function. This correction term was constructed following Szokoly et al. (2004). In addition, since BLL spectra are generally simple power laws, we used our objects to monitor second-order contamination and residual errors in the sensitivity function. In extreme cases, we fit an average deviation from power law across all objects in a night, and treated it as a correction to our spectrophotometric calibrations. This resulted in excellent, stable response functions for the major data sets.

Spectra were corrected for atmospheric extinction using standard values. We followed Krisciunas et al. (1987) for WMKO LRIS spectra, and used the mean KPNO extinction table from IRAF for P200 DBSP spectra. Our NTT, VLT, SOAR, and HET spectra do not extend into the UV and so suffer only minor atmospheric extinction. These spectra were also corrected using the KPNO extinction tables. We removed Galactic extinction using IRAF's de-reddening function and the Schlegel maps
(Schlegel et al. 1998). We made no attempt to remove intrinsic reddening (i.e., from the host galaxy).

Telluric templates were generated from the standard star observations in each night, with separate templates for the oxygen and water line complexes. We corrected separately for the telluric absorptions of these two species. We found that most telluric features divided out well, with significant residuals only apparent in spectra with high $\mathrm{S} / \mathrm{N}$. On the HET spectra, residual second-order contamination prevented complete removal of the strong water band redward of $9000 \AA$.

When we had multiple epochs of these final cleaned, fluxcalibrated spectra with the same instrumental configuration, we checked for strong continuum variation. Spectra with comparable fluxes were then combined into a single best spectrum, with individual epochs weighted by $\mathrm{S} / \mathrm{N}$.

Due to variable slit losses and changing conditions between object and standard star exposures, we estimated that the accuracy of our absolute spectrophotometry is $\sim 30 \%$ (Healey et al. 2008), although the relative spectrophotometry is considerably better.

\subsection{General Trends}

To illustrate the principal trends in the BLL spectra we refer the reader to Figure 1. By definition, the dominant component is a power law. J0516-6207, however, shows that after removal of a power-law weak but broad $\mathrm{C}$ IV, C III, and $\mathrm{Mg}$ II features may occasionally be seen in high $\mathrm{S} / \mathrm{N}$ spectra. Here the equivalent widths $(<1 \AA)$ are sufficiently small to secure the identification as a BLL. However, should the continuum fade by $\sim 10 \times$, this would be classified (at that epoch) as an FSRQ. Such transition objects support the idea of a blazar continuum, rather than two distinct populations (Fossati et al. 1998; Ghisellini \& Tavecchio 2008). In S12, we reported significant broad-line measurements for five of our BLLs, including J0516-6207.

While the power laws of most BLL are very blue, like J0516-6207, a few like J1849+2748 appear intrinsically flat or red, even after correcting for Galactic extinction. This may plausibly be a sign of a synchrotron component peaking near the optical, but might also indicate incomplete extinction correction, with residual reddening caused by dust not in the Schlegel et al. (1998) maps. It could also be intrinsic host extinction.

The Galactic reddening can be very severe. For J2001+4352 (upper right) we show both the highly extincted, pre-correction spectrum and the blue post-correction power law. This source is 


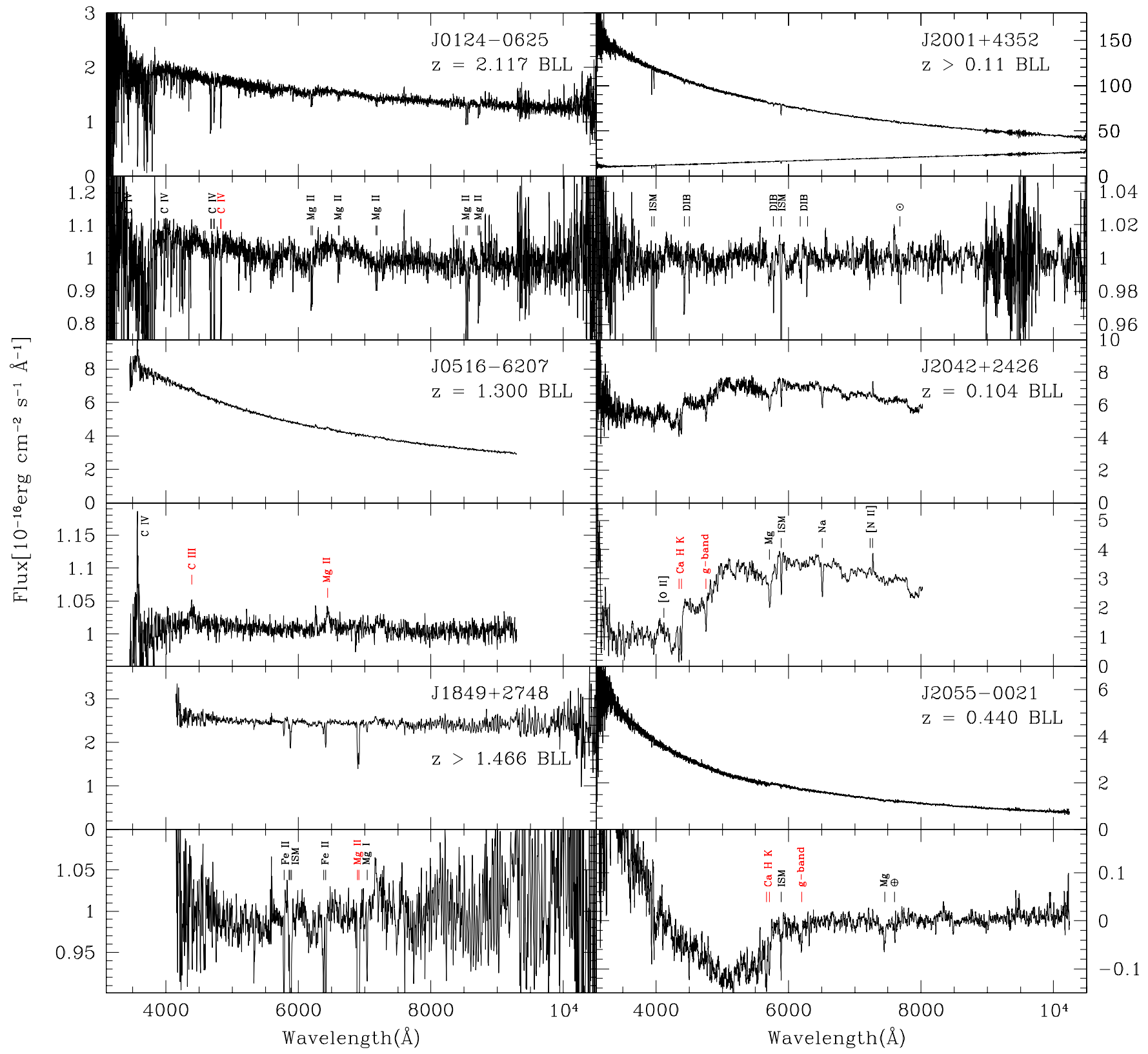

Figure 1. Spectra of Fermi BLLs. Each object is presented twice—directly in the upper panel, and then with the best-fit power law "removed" (generally by division, but for composite spectra by subtraction). This residual is plotted in the lower panel. SDSS spectra, while discussed in Table 3, are not replotted in this figure. The six sample spectra here illustrate major trends. Similar figures for the full BLL sample are available in the online journal. There we mark only the two lines used to secure $z_{\text {spec }}$; here, other lines of interest are marked and the two qualifying lines are marked in red. $z$ limits given are the most constraining limits presented-2 digits are given for limits from non-detection of host galaxies; 3 digits, for intervening absorption systems. The marked absorption system is the best spectroscopic limit; in some cases, a stronger host galaxy limit is presented. See Table 3 for the precise spectroscopic limit.

(The complete figure set (50 images) and a color version of this figure are available in the online journal.)

in a direction of known high $A_{V}=1.75$. Such extincted powerlaw spectra provide an excellent opportunity for interstellar medium (ISM) studies: the features seen after de-extinction and division by the best-fit power law (lower panel) are all interstellar in origin-Galactic $\mathrm{H}$ and $\mathrm{K}$, Na I 5892, and a series of diffuse interstellar bands, as described in Yuan \& Liu (2012).

For J0124-0625 (upper left) the residual absorption features are intergalactic in origin. Redward of $3900 \AA$ we detect a number of metal-line systems, blueward one sees the onset of strong Ly $\alpha$ forest absorptions. These features determine a redshift $z=2.117$ (one of the highest in our BLL sample). The lack of similar Ly $\alpha$ forest absorption in many of our other
high-S/N, high-resolution spectra allows us to place statistical upper limits on the redshift as described in Section 3.3.

Finally, at the lower right we see two BLLs with significant flux from the host. In J2042+2426, the galaxy provides about a third of the total flux and is easily visible in the raw spectrum. This is still safely a BLL, and we can measure the continuum contribution by a "Non-Thermal Dominance" (see Section 5, here NTD = 1.38). For J2055-0021, the host is swamped by the core synchrotron emission $(\mathrm{NTD}=47.5)$ and the galaxy features are visible only after subtracting the best-fit power law, as in the lower right panel. The flux increase in the blue appears due to residual few percent fluxing errors (here, likely 
incomplete correction for atmospheric extinction), rather than intrinsic emission. Despite the careful calibration, such residual fluxing issues persist in several spectra. However, the high-pass filtering described in Section 3.4 ensures that our measurements of, and bounds on, host galaxy flux are almost completely immune to such residual calibration artifacts. We find that a number of BLLs show visible host galaxy components, all consistent with giant ellipticals (Urry et al. 2000). We discuss the flux distribution of these host galaxies in Section 3.7.

\subsection{Individual Objects}

A number of BLL reductions required special treatment. For example, a few objects clearly required changes to the Schlegel map $A_{V}$, so that the de-extinction resulted in clean power laws. For $\mathrm{J} 0007+4712, A_{V}$ was increased from 0.3 to 0.8 and for J1941-6211 from 0.3 to 1.0. Conversely, we decreased the $A_{V}$ of $\mathrm{J} 1603-4904$ from 7.8 to 5.0 and $\mathrm{J} 2025+3343$ from 6.15 to 5.0. We checked the recent recalibration of the Galactic extinction maps (Schlafly \& Finkbeiner 2011), but did not find large changes, so these extinction features affecting our BLL are probably on scales below the map resolution.

$\mathrm{J} 1330+7001$ was observed off of the parallactic angle- the ensuing drop in blue flux is not intrinsic to the system, and our power law is fit only redward of $5000 \AA$. We thus remove the broadband residual in our power-law-divided spectrum in Figure 1. J1829+2729 and a nearby star were spatially unresolved in our data. The presented spectrum is a composite of starlight and quasar light, which the significant emission features all identified as $z=0$ stellar or ISM features.

In a few cases, objects previously cataloged as BLLs do not have sufficient $\mathrm{S} / \mathrm{N}$ in our spectra for a definitive BLL classification. For J0801+4401, we find that undetected broad lines could have an equivalent width as large as $9.5 \AA$; for J0209-5229 the limit is $5.6 \AA$, for J1311+0035 the limit is $8.0 \AA$, and for $\mathrm{J} 1530+5736$ we could have missed lines as strong as $\mathrm{EW}=5.5 \AA$. As higher $\mathrm{S} / \mathrm{N}$ spectroscopy would likely confirm the archival BLL designations, we consider them BLLs for the purposes of this paper.

Five of the BLLs described here had high significance broadline detections and have already been described in S12; we remeasure these spectra here for a uniform BLL treatment. In J0847-2337 and J0430-2507, the flux and spectral index measurements differ from the $\mathrm{S} 12$ values. This is because in the present analysis we first subtract the host galaxy flux, and calculate the flux and spectral index of the remaining non-thermal component. In S12, no such correction was attempted.

\section{MEASURING BLL REDSHIFTS}

The opportunity to advance our understanding of BLL evolution with the large, flux-limited Fermi sample is important (Ajello et al. 2012). Yet, despite the substantial telescope resources and careful analysis summarized above, many BLLs did not yield direct spectroscopic redshifts due to the extreme weakness of their emission lines (Sbarufatti et al. 2005b) and lack of clear host features. Therefore, we collect here both the direct redshift measurements and quantitative constraints on the allowed redshift range for our observed BLLs.

\subsection{Emission Line Redshifts}

We visually inspected all spectra for AGN emission-line features, and host galaxy absorptions. Spectroscopic redshifts are measured by cross-correlation analysis using the rvsao package (Kurtz \& Mink 1998). We require one emission line to be present at the $>5 \sigma$ level, and a second line present at the $>3 \sigma$ level-significances are measured by fitting a Gaussian template in the splot tool; we allow the width and amplitude of the Gaussian to vary, but fix the center at the redshift derived by rvsao's xcsao routine. For this study, we limited our search to typically strong emission lines known to be present in some BL Lacs: broad emission from C IV (1549, $1551 \AA$ ), C III (1909 ^), Mg II (2796, 2799, $2804 \AA), H \gamma(4340 \AA), H \beta$ (4861 $\AA$ ), and $\mathrm{H} \alpha(6563 \AA)$ and narrow emission from [O II] (3727, $3729 \AA)$, [O III] (4959, $5007 \AA$ ), and [N II] $(6549,6583 \AA)$. While other species exist in our spectra, these here listed are sufficient for definite redshift IDs. Velocities are not corrected to heliocentric or LSR frames.

In many cases, spectroscopic redshifts are further determined by a significant $(>3 \sigma)$ detection of a host galaxy, as will be described in Section 3.4. A few redshifts require further note: In J0124-0625 and J1451+5201, we identify the redshift by a Ly $\alpha$ and C IV absorption system at the onset of the Ly $\alpha$ forest. In J0434-2015, we identify a single strong feature with [O II], consistent with weak $\mathrm{Mg}$ II and $\mathrm{Ca} \mathrm{H} / \mathrm{K}$ absorptions. For $\mathrm{J} 1728+1215$, we find strong Mg II, confirmed by [O II] at the same $z$ in an archival spectrum. In $\mathbf{J} 2152+1734$, we identify a strong feature with $\mathrm{Mg}$ II confirmed by a significant [O II] detection in archival spectroscopy.

For a few objects only a single emission line was measured with high $\mathrm{S} / \mathrm{N}$. In general, we use the lack of otherwise expected features to identify the species and the redshift with high confidence. Nevertheless, a few redshifts have some systematic uncertainty and are marked by a ":" in Table 3. We briefly discuss these cases here. For J0007+4712, we derive a redshift from the clear onset of the $\operatorname{Ly} \alpha$ forest and report only two significant figures. In J0212+2244, we determine a tentative $z$ from weak Ca H, K, and $g$-band absorptions. For J0439-4522, we identify the one strong emission feature as C IV; intervening absorption excludes a Mg II identification, but a less likely C III identification at $z \sim 1.45$ is not conclusively ruled out. J0629-1959 presents broad but weak emission at the redshift of the highest $z$ metal line absorption system (1.724). We thus tentatively identify this as the object's true $z$. For J0709-0255, we identify the strong feature at $9200 \AA$ with [O III] by the line shape; an $[\mathrm{O} \mathrm{II}]$ identification at $z \sim 0.84$ is not excluded. For J0825+0309, we find significant [O III] emission at $5007 \AA$ (and possible but not significant emission at $4959 \AA$ ), at a $z$ consistent with an $\mathrm{Mg}$ II feature identified in Stickel et al. (1993). We find weak features in J1231+2847 at the SDSS $z$, but they have low significance. For J1312-2156, we find a plausible $\mathrm{Mg}$ II feature; this single line identification is in a small allowed redshift range $(z \sim 1.6)$; other identifications for this line are spectroscopically excluded. In J1754-6423, we tentatively identify emission at $\sim 6300 \AA$ with $\mathrm{Mg}$ II- higher $z$ redshifts are excluded by the lack of Ly $\alpha$ forest. In J2116+3339's spectrum, a significant broad emission feature is identified with C IV, consistent with a weak bump in the far blue at Ly $\alpha$. Nevertheless, a lower $z$ redshift is possible if the purported Ly $\alpha$ line is not real. J2208+6519 presents one strong, broad emission feature, tentatively identified as $\mathrm{Mg} \mathrm{II}-\mathrm{a} \mathrm{C}_{\mathrm{IV}}$ identification at $z \sim 1.8$ is not excluded.

\subsection{Intervening Absorbers}

For some BLLs, the core light passes near an intervening galaxy on its way to Earth. At small radii one can encounter low 
BLL Spectral Properties

\begin{tabular}{|c|c|c|c|c|c|c|c|c|c|c|c|c|c|c|c|c|}
\hline 2FGL & R.A. & Decl. & $\begin{array}{l}\text { Name } \\
\end{array}$ & $\begin{array}{l}\log F_{v, 10^{14.7}} \\
{ }^{28} \mathrm{erg} \mathrm{cm}^{-2} \mathrm{~s}\end{array}$ & $\left.\mathrm{~Hz}^{-1}\right)^{\alpha}$ & $M_{R}$ & $z$ & $\mathrm{ID}^{\mathrm{a}}$ & $z_{\min }{ }^{b}$ & $\begin{array}{c}z_{\min } \\
(-22.5)\end{array}$ & $\begin{array}{c}z_{\min } \\
(-22.9)\end{array}$ & $z_{\max }$ & Type & SED & Tel. & MJD \\
\hline J0000.9-0748 & 0.325017 & -7.774111 & J0001-0746 & $1.55 \pm 0.11$ & $-1.399 \pm 0.003$ & $\ldots$ & $\ldots$ & r. & $\ldots$ & 0.33 & 0.50 & 1.84 & BLL & IBL & P200 & 55064 \\
\hline $\mathrm{J} 0007.8+4713$ & 1.999862 & 47.202135 & $\mathrm{~J} 0007+4712$ & $1.33 \pm 0.14$ & $-1.431 \pm 0.430$ & $\ldots$ & 2.1: & $\mathrm{S}$ & 1.659 & 0.26 & 0.36 & 2.69 & BLL & LBL & P200 & 55743 \\
\hline J0009.0+0632 & 2.267006 & 6.472664 & $\mathrm{~J} 0009+0628$ & $1.38 \pm 0.11$ & $-1.410 \pm 0.007$ & $\ldots$ & $\ldots$ & . & $\cdots$ & 0.58 & 0.76 & 1.65 & BLL & LBL & WMKO & 55040 \\
\hline $\mathrm{J} 0009.1+5030$ & 2.344750 & 50.508005 & $\mathrm{~J} 0009+5030$ & $1.51 \pm 0.11$ & $-0.776 \pm 0.010$ & $\ldots$ & $\ldots$ & . & $\ldots$ & 0.79 & 0.79 & 1.64 & BLL & $\ldots$ & WMKO & 55475 \\
\hline J0012.9-3954 & 3.249804 & -39.907184 & J0012-3954 & $1.01 \pm 0.11$ & $-1.248 \pm 0.032$ & $\ldots$ & $\ldots$ & - & $\ldots$ & 0.52 & 0.64 & 2.52 & BLL & $\ldots$ & VLT & 54362 \\
\hline J0013.8+1907 & 3.485099 & 19.178246 & $\mathrm{~J} 0013+1910$ & $0.61 \pm 0.11$ & $-2.250 \pm 0.076$ & $\ldots$ & 0.477 & B & $\ldots$ & 0.41 & 0.54 & 2.17 & BLL & $\ldots$ & WMKO & 54470 \\
\hline J0018.5+2945 & 4.615626 & 29.791743 & J0018+2947 & $1.13 \pm 0.11$ & $-0.701 \pm 0.181$ & $\ldots$ & $\ldots$ & . & $\ldots$ & 0.89 & 0.94 & 1.71 & BLL & HBL & WMKO & 55547 \\
\hline J0018.8-8154 & 4.841042 & -81.880833 & J0019-8152 & $1.73 \pm 0.12$ & $-0.975 \pm 0.045$ & $\ldots$ & $\ldots$ & . & $\ldots$ & 0.24 & 0.34 & 2.32 & BLL & HBL & NTT & 55778 \\
\hline J0021.6-2551 & 5.385517 & -25.846999 & $\mathrm{~J} 0021-2550$ & $1.63 \pm 0.11$ & $-0.689 \pm 0.005$ & $\ldots$ & $\ldots$ & . & 0.564 & 0.57 & 0.58 & 1.63 & BLL & IBL & WMKO & 55476 \\
\hline J0022.2-1853 & 5.538158 & -18.892486 & J0022-1853 & $1.94 \pm 0.11$ & $-1.061 \pm 0.069$ & $\ldots$ & $\ldots$ & . & 0.774 & 0.13 & 0.15 & 1.64 & BLL & HBL & WMKO & 55476 \\
\hline J0022.5+0607 & 5.635264 & 6.134573 & $\mathrm{~J} 0022+0608$ & $1.86 \pm 0.11$ & $-1.560 \pm 0.009$ & $\ldots$ & $\ldots$ & . & $\ldots$ & 0.29 & 0.42 & 1.63 & BLL & LBL & WMKO & 55060 \\
\hline J0029.2-7043 & 7.170792 & -70.754694 & J0028-7045 & $1.38 \pm 0.11$ & $-1.139 \pm 0.011$ & $\cdots$ & $\ldots$ & . & 0.966 & 0.54 & 0.58 & 1.95 & BLL & .. & VLT & 55056 \\
\hline J0033.5-1921 & 8.393060 & -19.359393 & J0033-1921 & $1.94 \pm 0.12$ & $-0.852 \pm 0.053$ & $\ldots$ & $\ldots$ & . & $\cdots$ & 0.29 & 0.37 & 1.77 & BLL & HBL & P200 & 55567 \\
\hline J0035.2+1515 & 8.810913 & 15.250758 & $\mathrm{~J} 0035+1515$ & $1.93 \pm 0.11$ & $-0.895 \pm 0.003$ & $\ldots$ & $\ldots$ & . & $\ldots$ & 0.48 & 0.48 & 1.65 & BLL & HBL & WMKO & 55357 \\
\hline J0037.8+1238 & 9.461902 & 12.638514 & $\mathrm{~J} 0037+1238$ & $2.00 \pm 0.11$ & $-1.163 \pm 0.010$ & $-22.4 \pm 0.1$ & 0.089 & G & $\cdots$ & 0.09 & 0.09 & 1.76 & BLL & HBL & WMKO & 55357 \\
\hline
\end{tabular}

Notes.

${ }^{a}$ Method for $z$ ID—B: broad emission lines; N: narrow emission lines; G: host galaxy features; S: special case—see Section 3.1.

${ }^{\mathrm{b}}$ Spectroscopic lower limits (i.e., from intervening absorption systems).

(This table is available in its entirety in a machine-readable form in the online journal. A portion is shown here for guidance regarding its form and content.) 
excitation clouds in the galaxy's halo, giving absorption doublets from Mg II at $(2795.5,2802.7) \AA$. Larger impact parameters can sample C IV at $(1548.2,1550.77) \AA$. In some low excitation (i.e., Mg II) systems, we also see absorption from Fe II at (2344.2, 2374.4, 2382.7, 2586.6, 2600.1) ^. Finally, for our highest redshift BLL we Ly $\alpha$ absorption at $1215 \AA$ for the metal line systems, as well as onset of the Ly $\alpha$ forest.

For unsaturated absorptions, the doublet ratio for $\mathrm{Mg}$ II and $\mathrm{C}$ IV is $2: 1$, with the blue line dominant. In saturated absorptions, the ratio is 1:1 (Nestor et al. 2005; Michalitsianos et al. 1988).

We visually search all spectra for candidate doublets, and follow Nestor et al. (2005) in employing a quantitative test of the significance of each candidate. We used a two Gaussian template with wavelength spacing scaling with $z$, but free amplitudes, and fit the equivalent width and error of each component, using the splot tool in IRAF. For the candidate to qualify as a detection we require the stronger (bluer) line to have $>5 \sigma$ significance, and the second line to have $>3 \sigma$ significance. In a few cases, one component of an otherwise strong doublet was affected by skylines, telluric features, or cosmic rays. In these cases, another expected feature from the absorption complex (e.g., a Fe II line) detected at $>3 \sigma$ qualified the system. We further require the doublet ratio to be consistent (within errors) to a value between $2: 1$ and $1: 1$.

Our principal goal is not an absorption line study. Thus, we concentrated on the longest wavelength (highest $z$ ) candidate system and measured sufficient lines to confirm the $z$ (i.e., two significant lines). After validation we continued to search for higher $z$ until no candidates passed the significance test. We therefore believe that we have found the highest $z$ absorption system in each of our spectra strong enough to reach the $5 \sigma / 3 \sigma$ criteria above.

Since we see the onset of the Ly $\alpha$ forest in our highest redshift objects, the red end of the forest provides a strict lower limit on redshift. This can be higher than that inferred from the reddest metal line system.

We list these spectroscopic minimum $z \mathrm{~s}$ as $z_{\min }$, when available, in Table 3 .

\subsection{Redshift Upper Limits}

We can use the absence of $\operatorname{Ly} \alpha$ absorptions to provide statistically based upper limits on $z$ for all BLLs without redshift. The exclusion $z_{\max }$ depends on the spectral range, resolution, and $\mathrm{S} / \mathrm{N}$ of the particular observation, but is generally $1.65<$ $z<3.0$.

To quantify the upper bound, we need the expected density of $\operatorname{Ly} \alpha$ forest absorbers. Penton et al. (2004) find that for rest $\mathrm{EW} \geqslant 0.24 \AA, d N / d z \sim 40$ at $z=1.6$, varying with redshift as $\log d N / d z \propto 1.85 \log (1+z)$. We follow Weymann et al. (1998) for the EW scaling: $d N / d z \propto e^{-\left(\mathrm{EW}_{\text {rest }}-0.24\right) / 0.267}$. As the $\mathrm{S} / \mathrm{N}$ and resolution of our data vary, we generally measured a conservative uniform $3 \sigma$ sensitivity for narrow Ly $\alpha$ absorptions $100 \AA$ from the blue end of our spectra. Typical EW limits in this range were $\sim 0.2-1.0 \AA$. Given this density we solve for the $\Delta z$ range giving a Poisson probability of 0.32 (i.e., $1 \sigma$ ) for detecting no absorbers, obtaining

$$
\Delta z=0.167 e^{\left.\left(\mathrm{EW}_{\mathrm{rest}}-0.24\right) / 0.267\right)}\left(\frac{\lambda_{\min }}{1215}\right)^{-1.85},
$$

where $\lambda_{\min }$ is the effective blue end of the spectrum (generally $3150-4200 \AA$ ), and EW is the measured equivalent width limit. Thus, we infer a maximum source redshift $z_{\max }=$ $\left(\lambda_{\min } / 1215\right)-1+\Delta z$. In some cases, the $\mathrm{S} / \mathrm{N}$ is too low at the blue end of the spectrum. We then measure EW limits closer to the sensitivity peak of the spectrum. Of course, with a larger $\lambda_{\text {min }}$ for the effective spectrum end Equation (1) gives less constraining upper limits.

If the actual blazar redshift $z$ is very close to $z_{\max }$ as estimated above, then its UV radiation may photoionize Ly $\alpha$ clouds along the line of sight, postponing the onset of the forest and artificially lowering $z_{\max }$. In practice, the effect is usually small $\left(\Delta z_{\max } \sim 0.01-0.02\right)$ except for large $z_{\max }$ when our bound is generally not of interest. We follow Bajtlik et al. (1988) to estimate this "proximity effect" correction, by computing

$$
\omega\left(z_{\mathrm{Ly} \alpha}\right)=\frac{f_{v}}{4 \pi J_{v}} \frac{\left(1+z_{\mathrm{Ly} \alpha}\right)^{5}}{(1+z)}\left[\frac{(1+z)^{1 / 2}-1}{(1+z)^{1 / 2}-\left(1+z_{\mathrm{Ly} \alpha}\right)^{1 / 2}}\right]^{2},
$$

where $f_{v}$ is the blazar flux at the absorption Lyman limit (at $z_{\text {Ly } \alpha}$ ) and $J_{v}=10^{-21.5} \mathrm{erg} \mathrm{cm}^{-2} \mathrm{~s}^{-1} \mathrm{~Hz}^{-1} \mathrm{sr}^{-1}$ is the cosmic ionizing flux, conservatively estimated for our redshift range. We compute $f_{v}$ from the power-law fit in Table 3, and increase the blazar redshift $z$ until $\omega\left(z_{\mathrm{Ly} \alpha}\right)<1$. We thus adopt these revised $z_{\max }=z$ and quote these corrected upper limits in Table 3.

\subsection{Host Galaxy Fitting}

It has been claimed that BL Lac objects are hosted by giant elliptical galaxies with bright absolute magnitude $-M_{R}=$ $-22.9 \pm 0.5$ in our cosmology (Urry et al. 2000; Sbarufatti et al. 2005b).

If we adopt the common assumption that these are standard candles, we can estimate the redshift of the BLL by detecting such galaxies. In imaging studies, one looks in the wings of the BLL for the host galaxy flux, and compares that to the standard candle flux at various redshifts (Sbarufatti et al. 2005a; Meisner \& Romani 2010). In spectroscopic studies, one typically looks for individual absorption features (i.e., H, K, g-band). One can also use the lack of such lines as evidence that the BLL is at higher redshift (Sbarufatti et al. 2005b; Shaw et al. 2009).

With high $\mathrm{S} / \mathrm{N}$ spectra, however, one can obtain more stringent limits by using the entire elliptical template rather than just one (or a few) lines. Plotkin et al. (2010) developed a technique of fitting for host galaxies in SDSS BLL spectra. We expand here on that method for our more heterogeneous spectra.

Our spectra come from a variety of spectrographs in disparate observing conditions and we find low-frequency systematic fluctuations in many of the fits. These are likely caused by imperfect spectrophotometric fluxing and second-order contamination as discussed in Section 2.3. These effects can dominate over real galaxy features. Using SciPy's Signal Processing routines, ${ }^{4}$ we construct a bandpass Kaiser window from $220 \AA$ to $1.5 \times$ the Nyquist frequency. We apply that window as an effective highpass filter both to our spectra and to the templates to mitigate this low-frequency noise before fitting (Kaiser \& Schafer 1980).

We test possible redshifts $z_{i}$ on a grid scaled to the spectrograph resolution with constant spacing in $\log z$. This grid is thus $z_{i}=\left(\left(2 \Delta \lambda / \lambda_{0}\right)+1\right)^{i}-1$, where $\Delta \lambda$ is the pixel scale of the spectrograph. For each trial $z_{i}$ we fit the power law $F_{v} \propto v^{\alpha}$ index and flux and the amplitude of a redshifted elliptical template. This host template is generated from the PEGASE model (Fioc \& Rocca-Volmerange 1997) tables and evolved to low

\footnotetext{
4 Documentation and more information available at http://docs.scipy.org/doc/scipy/reference/signal.html.
} 

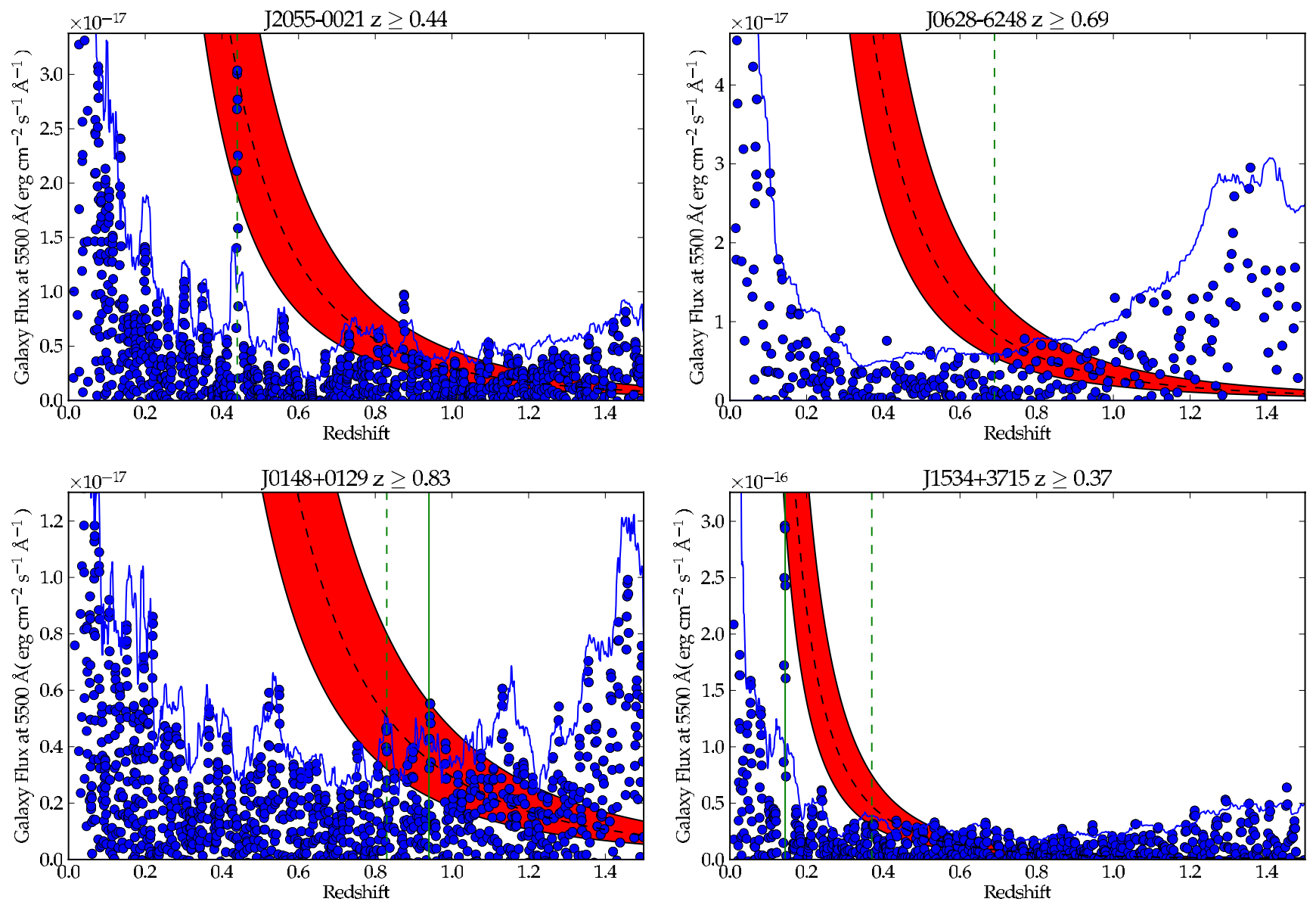

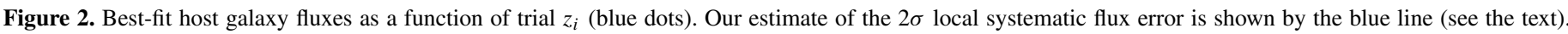

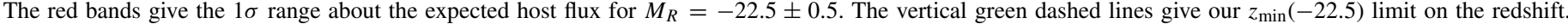

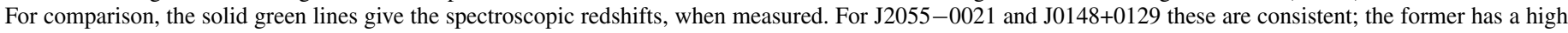

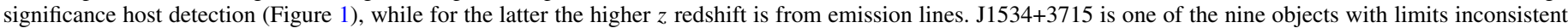
with spectroscopic redshifts. Here, the host galaxy is subluminous $\left(M_{R}=-21.87 \pm 0.16\right)$ and thus (just) missed by this technique (Prob $\left.=0.15\right)$.

(A color version of this figure is available in the online journal.)

$z$ from $z=2$, as in O'Dowd \& Urry (2005). For uniformity, we here use the same template for all $z_{i}$, and do not perform any evolution corrections.

Our fit minimizes $\chi^{2}$ with three free variables at each trial $z_{i}$. We employ the scipy.optimize.leastsq routine based on a Levenberg-Marquardt fitter.

To model host slit losses, we assume an $r=10 \mathrm{kpc}$ de Vaucouleurs profile with Sérsic index 1/4 (O'Dowd \& Urry 2005) and account for the individual observations' slit width and seeing profile (measured from the core FWHM). Since we have employed optimal extractions of the BLL spectra, our effective aperture along the slit varies, but we estimate a typical width of $\approx 2 \times$ the spatial FWHM achieved during our spectral integration. Accordingly, we estimate host slit losses through a rectangular aperture of the slit width $\times$ twice the spectrum FWHM. Inferred host fluxes are corrected for these slit losses.

Results of sample fits are shown as the blue dots in Figure 2 where the fit amplitude of the host galaxy template is plotted against trial redshift.

\subsection{Power-law Fit}

We report the power-law fluxes and spectral indices of the best fit to the de-extincted spectrum in Table 3 . The flux is given in units of $\log 10^{-28} \mathrm{erg} \mathrm{cm}^{-2} \mathrm{~s}^{-1} \mathrm{~Hz}^{-1}$ as observed at $10^{14.7} \mathrm{~Hz}(\sim 5980 \AA)$, the center of our typical spectral range. The index $\alpha$ is measured $F_{\nu} \propto v^{\alpha}$. These values may be combined with multi-wavelength data to study the continuum SED of the blazars in our sample. Since the statistical errors on the fit are, in general, unphysically small, we follow S12 in estimating errors on the spectral index by independently fitting the red and blue halves of the spectrum. Note that large error bars generally indicate a relatively poor fit to a simple power law rather than large statistical errors. The statistical errors on the $F_{v}$ amplitude are also small; we convolve these with our estimated 30\% overall fluxing uncertainty (Healey et al. 2008), which dominates in nearly all cases.

For objects with high significance $(>3 \sigma)$ detections of galaxies, as described in Section 3.7, we report the best-fit power law from the simultaneous power-law/host fit in Section 3.4.

When we have observed objects at multiple epochs, we also fit a power law to the other, non-primary spectra. These fluxes vary substantially, some by more than $10 \times$. In Figure 3, we show the distribution of $f_{\max } / f_{\min }$ flux ratios. This is well described by an $\alpha=1.29$ power law. Epochs from our fiducial spectra are listed in Table 3.

\subsection{Testing the Standard Candle Assumption}

BLL with a redshift and a secure $(>3 \sigma)$ host detection can be used to test the uniformity of the host luminosities. There are 59 such BLLs in our sample. We derive synthetic $R$-band 


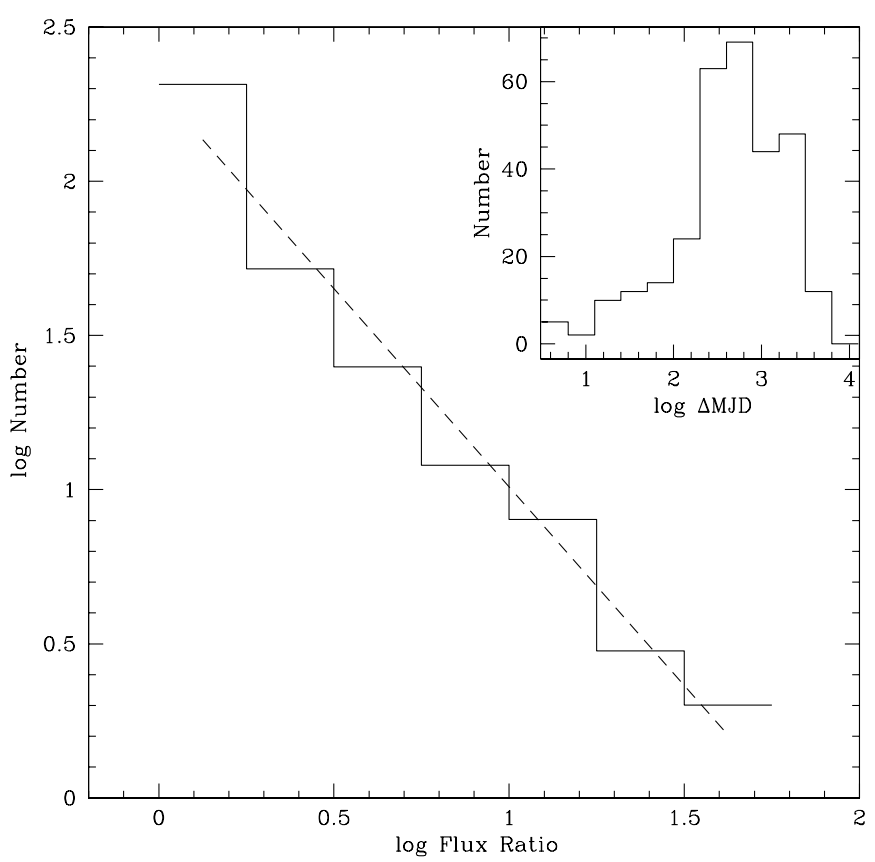

Figure 3. Histogram of maximum flux ratios. For each object where we have collected multiple spectra, we compute the flux ratio between the power-law components of the brightest and faintest observed epoch. These are plotted as a $\log -\log$ histogram. The dashed line is the best-fit power law with index $\alpha=1.29$. The inset histogram shows the distribution of time between observations - typically $\sim 1$ year.

magnitudes by applying a Kron-Cousins $R$ filter to our spectra (Meisner \& Romani 2010). The results are shown as a histogram in Figure 4. We find $\left\langle M_{R}\right\rangle=-22.5$, down $\sim 0.4$ mag from $M_{R}=-22.9$ found in Sbarufatti et al. (2005b). We find a similar spread in luminosity ( $\sim \pm 0.5 \mathrm{mag})$. When we separate the host measurements for lower-peak sources (LBL+IBL) we find that they have a median luminosity $\sim 0.3$ mag fainter than that of our HBL. Unfortunately, we do not have sufficient LBL+IBL hosts to test for such differences at high significance. Past studies differ: Urry et al. (2000) found no significant offset in the host magnitudes of HBL and LBL, but Sbarufatti et al. (2005b) noted that higher peak HBL tend to have more luminous hosts.

Two of the high significance host galaxies have imaging magnitudes reported in Sbarufatti et al. (2005b). For J1442+1200, we measure $M_{R}=-22.99 \pm 0.14$; Sbarufatti et al. (2005b) reported $M_{R}=-22.77$. For J1428+4240, we measure $M_{R}=$ $-22.78 \pm 0.13$; Sbarufatti et al. (2005b) find $M_{R}=-22.75$. These are consistent within measurement errors, a good check of our slit-loss corrections and magnitude estimates.

Overall, the LAT BLL sample thus represents a fainter host population of than those studied in previous work. Conceivably, a higher (LBL+IBL)/HBL ratio in our sample causes part of the difference (although we remain HBL dominated). However, we suspect that our rather exhaustive $8 \mathrm{~m}$ class campaign, skipping most objects with redshifts already in the literature, selects for fainter host galaxies than in the past. Thus, we may be probing fainter on the true host luminosity distribution; the BLL for which we were not able to provide host detections may then be similarly underluminous compared to previous studies. A true evaluation of the intrinsic host luminosity distribution, as well as any dependence on subclass type, will require a careful assessment of the parent population ( $\gamma$-ray) and host detection selection biases.

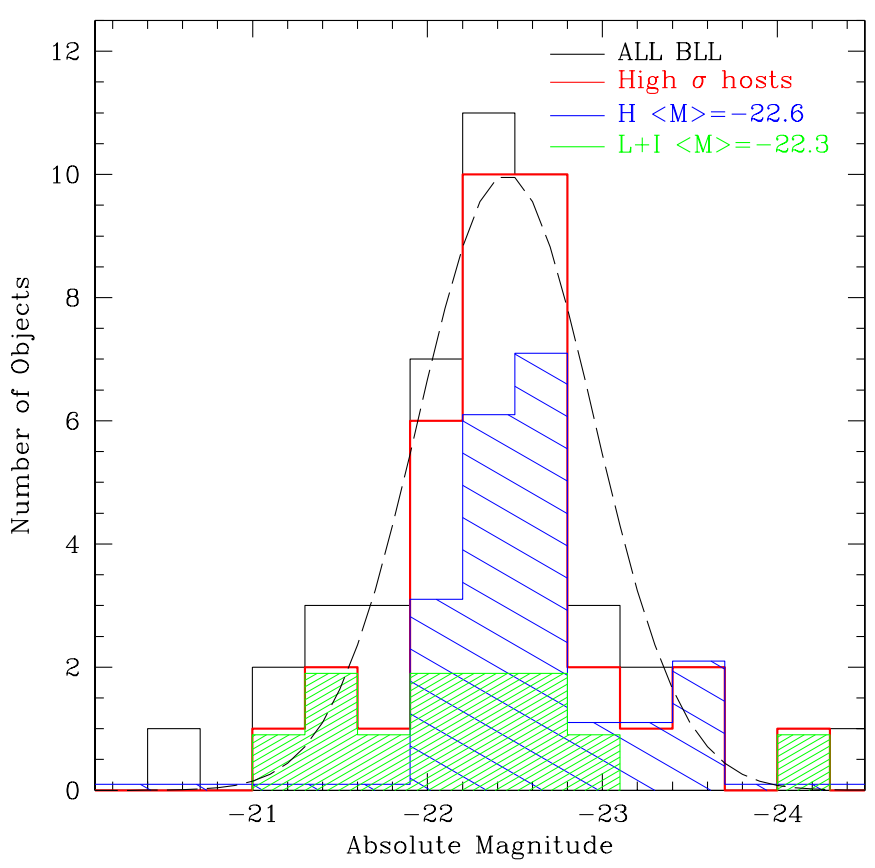

Figure 4. Measured BLL host absolute magnitudes ( $R$ equivalent at $z=0$ ). Black histogram: all BLL hosts with spectroscopic redshift. A weighted $\chi^{2}$ fit gives $M_{R}=-22.5 \pm 0.5$, our best estimate for the luminosity of BLL host galaxies. Red: hosts with high significance $(>3 \sigma)$ detections. The green and blue sub-histograms show high significance LBL+IBL and HBL hosts, respectively. (A color version of this figure is available in the online journal.)

In the rest of this section, we conservatively adopt our $M_{R}=-22.5 \pm 0.5$ estimate. We do also report (Table 3) more aggressive redshift limits based on the common assumption $M_{R}=-22.9 \pm 0.5$, for more direct comparison to previous work, but we recommend adoption of the less stringent $M_{R}=$ $-22.5 \pm 0.5$ redshift constraints.

\subsection{Lower Limits from Non-detections of Host Galaxy}

We use the results of the fitting in Section 3.4 and the calibration in Section 3.6 to constrain the redshift of the host. At each trial redshift, our fitter reports a flux $(f \pm \sigma f)$ for the host galaxy. This is to be compared with the model flux from the redshifted standard candle elliptical template $\left(f_{M} \pm \Delta f_{M}\right)$.

While we have greatly decreased the effect of the lowfrequency noise in our spectral fits using the high-pass filter, we still find that the statistical errors on the fit host galaxy fluxes are unrealistically small; these flux estimates remain dominated by systematic effects. We therefore construct an error estimate $\Delta f$ for each $z_{i}$ by measuring the dispersion of flux estimates for nearby redshift bins. This is computed from a sample of the 30 nearest $f(z)$, skipping 5 bins on each side of our test $z_{i}$ to minimize high-pass correlation. After $\sigma$-clipping, the fit flux distributions are well behaved and we use these to compute a $2 \sigma$ upper limit (scaled from the rms) at each $z_{i}$. The vectors of these upper limits are shown by the jagged blue lines in Figure 2. These have captured the local effective noise quite well and so we adopt these vectors as effective $2 \sigma$ confidence limits.

Fit fluxes well above these $2 \sigma$ limits denote likely host detections. Indeed, we found that this automatic processing was quite effective at flagging candidate $z_{i}$ for host detection. Here, however, we focus on how well a fit flux $f$ with local effective error $\Delta f$ can be used to exclude a host of the expected magnitude $f_{M}$ at the test redshift $z$. Thus we compute a probability that the fit flux $f$ and error $\Delta f$ are consistent with the expected model 


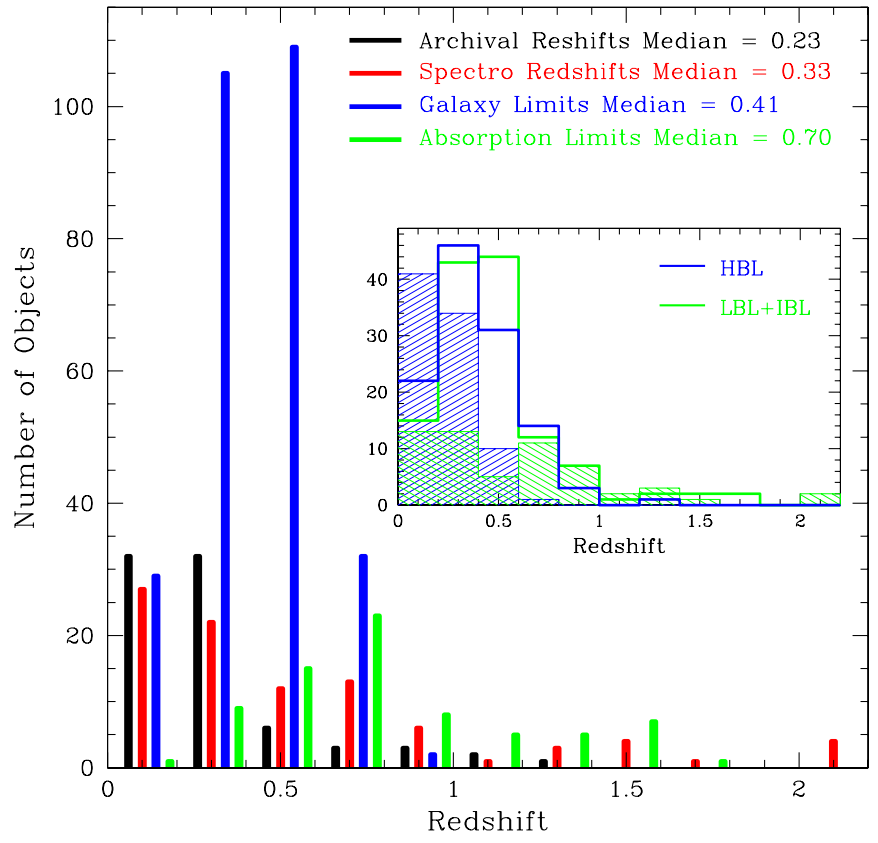

Figure 5. BLL redshift and redshift lower limit distributions. Within each $\delta z=0.2$ bin, we show (left to right) the archival $z$, our new spectroscopic $z$, lower limit $z$ s from host fitting, and lower limit $z$ s from intervening absorption line systems. The limits show that the spectroscopic redshift samples, particularly the archival sample, are selection biased to low $z$. The subframe shows the redshifts (filled histograms) and lower limits (open histograms) for the LBL+IBL (green) and HBL (blue). Again, both limit histograms extend to higher $z$. The LBL+IBL sample extends somewhat further than the HBL.

(A color version of this figure is available in the online journal.)

flux $f_{M}$ and its uncertainty at the given $z$ as

$$
\operatorname{Prob}(z ; f, \Delta f)=C \int_{f_{M}}^{\infty} G\left(f_{M}^{\prime}, \Delta f_{M}^{\prime}\right) G(f, \Delta f) d f_{M}^{\prime},
$$

where $G\left(x, \sigma_{x}\right)$ is a Gaussian of width $\sigma_{x}$ centered at $x$. The normalization $C$ is chosen such that Prob $=1$ for $f_{M}=0$ (i.e., any $f$ is acceptable for a model of zero flux). This is a conservative choice as it does not exclude overluminous hosts. For example, when we assume a model $M_{R}=-22.5 \pm 0.5$ this probability also finds any $f$ consistent with $M_{R}=-22.9 \pm 0.5$ to be acceptable. When the fitter returns an unphysical negative $f$, we evaluate the probability for $f=0$ and the local $\Delta f$.

This probability becomes substantial for $z$ near a good candidate redshift. It also grows as the sensitivity of our host search drops at large $z$. We thus calculate a minimum redshift $\left(z_{\min (-22.5)}\right)$ as the lowest redshift for which Prob $\geqslant 0.17$ (i.e., $1 \sigma)$. We list these values in Table 3 . For comparison, we also give $z_{\min (-22.9)}$ calculated in the same fashion, assuming a model $M_{R}=-22.9 \pm 0.5$. Comparison between the spectroscopic detections and $z_{\min }$ suggest that the $M_{R}=-22.5$ value is most consistent with observed detections, and lower bounds (as expected from Section 3.6). We recommend the use of these conservative lower limits. Note also that the vector $\operatorname{Prob}(z)$, once normalized with an appropriate prior and truncated at $z_{\max }$, can be used as a PDF for the BLL redshift.

\subsection{Redshift Distribution}

As seen in Figure 5, archival redshift measurements for BLL are dominated by low values $(\tilde{z}=0.23)$. Our new spectroscopic redshifts have extended the population to higher $z$, with some objects' redshifts at $z \gg 1$. Still, the objects with redshifts remain dominated by low $z$. In the new spectroscopic redshifts we find $\tilde{z}=0.33$. We believe there is a significant bias to low redshift in both of these samples, as the weak low EW emission or absorption features of our typical BLLs with known redshift are easier to detect at low $z$.

In Figure 5 we also show two sets of redshift lower limits. For every object in our sample, we can derive a host-detection limit $(\tilde{z}=0.41)$. These lower limits on redshift are still biased low: as evident from Figure 2 we are most sensitive to galaxies at low $z$. Nevertheless, they suggest that these objects are not consistent with the spectroscopic redshifts (the K-S test gives probability $<10^{-11}$ of consistency with archival redshifts). The absorption line limits we have for some objects (described in Section 3.2) give further evidence for a population of BLLs at high redshift $(z>1)$. Together, these results strongly imply that previous BLL studies suffered important biases due to shallow samples with large redshift incompleteness preventing detection of bright, but high $z$ BLL.

The inset shows the spectroscopic redshifts and the redshifts limits for high-peaked (HBL) and lower-peaked (LBL+IBL) sources classified in 2LAC. We see that the lower-peaked detections extend to higher $z$, as might be expected if these sources are more luminous and have a less dominant synchrotron continuum. However, the open histograms of limits remind us that both subclasses still suffer substantial redshift incompleteness, and the missing redshifts for both subclasses extend substantially higher than those in hand. A re-appraisal of the BLL population, properly including the new redshifts, $z$ constraints and remaining selection biases is needed to test whether either subclass is still consistent with negative cosmological evolution.

\section{BLACK HOLES AND HOST GALAXIES}

The masses of the central black holes provide important insight into the cosmic evolution of various AGN classes. These are most easily estimated by the virial technique (cf. Shen et al. 2011). In S12, we adopted this method to give mass estimates for the Fermi FSRQs. For BLL, the lack of high S/N broadline measurements precludes such estimates. However, we have measured a number of host magnitudes in Section 3.4; since these are ellipticals, this is all "bulge," and we can apply an $M-L$ relation to estimate the hole mass. We follow Gültekin et al. (2009):

$\log \left(\frac{M}{M_{\odot}}\right)=(8.95 \pm 0.11)+(1.11 \pm 0.18) \log \left(\frac{L_{V}}{10^{11} L_{\odot, V}}\right)$

where $M$ is the black hole mass and $L_{V}$ is the luminosity in a $V$ filter $\left(\log \left(L_{V} / L_{\odot, V}\right)=0.4\left(4.83-M_{V \text {, bulge }}^{0}\right)\right)$. To convert our fit template spectrum amplitudes to consistent $V$ magnitudes, we integrate our template spectrum over the Hubble F555W filter (Lauer et al. 2005) as in Section 3.6.

The masses from Equation (4) are plotted as circles in Figure 6. When the subclass is known (HBL or LBL+IBL) we fill these in with blue or green, respectively. For comparison, we show the $1 \sigma$ spread of virial-estimate $\mathrm{BH}$ masses from optically selected SDSS quasars from Shen et al. (2011, gray band) and masses of the Fermi FSRQs in S12 (red points). Of course, if BLL hosts really are standard candle ellipticals, then the $M_{\text {bulge }}-M_{\text {}}$ relation implies constant black hole masses. The masses corresponding to the standard $M_{R}=-22.9$ and our revised -22.5 are shown by dashed lines.

Interestingly, our BLL $M_{\text {• }}$ estimates increase with $z$ much as the optical QSO or FSRQ. Of course, we only plot high 


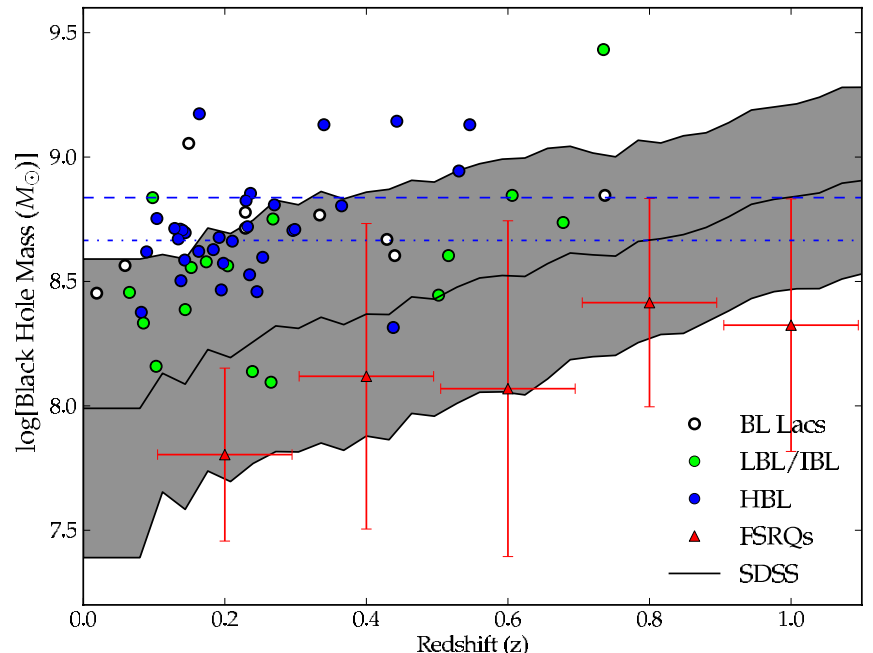

Figure 6. BLL black hole masses plotted as a function of redshift (circles; blue fill HBL, green fill LBL+IBL). The gray band shows the $1 \sigma$ spread of the optically selected SDSS black holes (Shen et al. 2011). Red error bars give the $1 \sigma$ spread Fermi FSRQ black hole masses (S12). The estimated BLL black hole masses are higher than for the two other AGN types. The upper (lower) blue lines show the black hole mass expected for the standard candle bulge at $M_{R}=-22.9(-22.5)$.

(A color version of this figure is available in the online journal.)

significance host detections here, and low-luminosity hosts at high $z$ are increasingly difficult to detect (unless the core luminosity decreases). Accordingly, as for the QSO, we suspect that the bulk of this trend is due to selection effects. In the case of the FSRQ, S12 argued that the offset to smaller black hole mass was at least partly due to a preferentially polar view of an equatorially flattened broad-line region, with the projection decreasing the observed kinematic line width and the average virial mass estimate. Like $\gamma$-ray-selected FSRQ, BLL are Doppler-boosted along our line of sight (Urry \& Padovani 1995). However, since the host flux is nearly isotropic, we expect

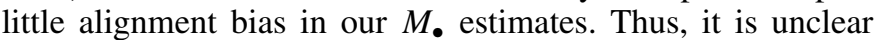
whether the BLL offset to larger black hole masses is real or selection dominated.

In a study of BLL hosts detected in the SDSS, León-Tavares et al. (2011) found no significant difference between the masses of the central black holes of HBL and LBL. In Figure 6 the HBL masses are, however, biased upward with respect to the lowerpeak BLL black hole masses. This is of course just a restatement of the offset in host luminosity seen in Figure 4. Unfortunately, we cannot claim that this is a physical difference as the trend is precisely what would expect from selection bias if HBL have brighter non-thermal cores.

Ideally, we could use these black hole masses to explore the relationship between the BLLs and the general QSO population. Large black hole masses, if not induced solely by selection bias, would imply a late stage of AGN evolution. The black hole mass is often compared to the source luminosity to characterize the state of the accretion in Eddington units. However, with the exception of the few BLL for which we see broad lines (which seldom have a significantly host detection), the observed flux is so dominated by beamed jet emission that quoting the accretion luminosity in Eddington units is not feasible.

\section{NON-THERMAL DOMINANCE}

In S12, we introduced the non-thermal dominance (NTD) as a quantitative measure of how much the optical is contaminated by

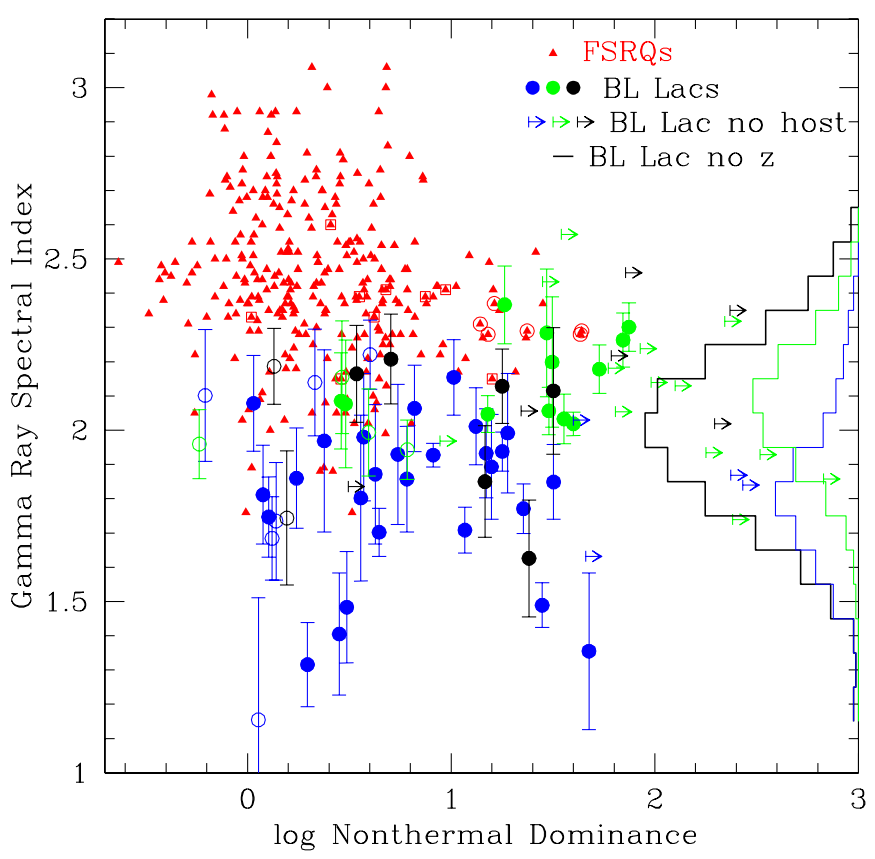

Figure 7. NTD plotted against $\gamma$-ray spectral index. The BLLs are plotted as dots; solid if $L_{\gamma}>10^{44}$; empty otherwise. Broad-line objects from S12 (typically FSRQs) are plotted as red triangles — circles surround those in a BLL state at the primary spectrum epoch; squares, those known to be BLL at a different epoch. BLLs with known $z$, but no thermal (host galaxy or broad emission line) detection are plotted as lower limits. BLLs without known $z$ are shown by the histogram at right. Blue symbols indicate HBL, green LBL+IBL. Except for the NTD increase for the BLL population relative to the FSRQ, no clear trend with spectral index is seen.

(A color version of this figure is available in the online journal.)

non-thermal synchrotron emission. We here extend that analysis to BLLs.

For most BLL, the dominant "thermal" contribution is the host galaxy, not the big blue bump. We therefore set NTD $\equiv$ $F_{\text {core }} / F_{\text {host }}$ where both fluxes are measured at $5100 \AA$-the same wavelength as the $\mathrm{H} \beta$ continuum measurements for FSRQs. The wavelength choice is important for BLL NTD measurements since the host galaxy is much redder than the continuum-dominated core; an NTD measurement just above (redward of) the $4000 \AA$ break would typically give values $\gtrsim 4 \times$ larger. Measurements below the break would, of course, diverge even more.

As noted, BLLs are highly variable so that their NTD changes over time. Our BLL can vary by over an order of magnitude in the optical (S12, Figure 3). In this study, our primary spectra for variable objects were generally drawn from the epochs giving the highest $\mathrm{S} / \mathrm{N}$ on weak emission or absorption features. As this favors low core fluxes, our primary epoch biases the results toward low NTD.

In Figure 7, we plot the NTD against $\gamma$-ray spectral index. The values for BLL with measured galaxy flux are plotted, along with lower limits for BLL with redshift, but no significant host detection. The histogram at the right shows the spectral index distribution of BLL with no redshift detection (and unknown NTD). We expect these BLLs to have higher NTD on average, as the larger core flux makes redshift determinations more challenging. When known, we indicate whether the BLLs are high-peaked sources (blue) or lower peaked (green). The FSRQs from $\mathrm{S} 12$ are plotted as red triangles.

The most striking trend in this figure is the vertical color separation. This is the well-known result that $\gamma$-ray spectral 
index hardens from FSRQ through LBLs to HBLs (Ackermann et al. 2011). We also see the defining characteristic of the BLL, increased continuum dominance with respect to the FSRQ. However, the NTD trend does not appear to continue through the BLL: harder spectrum BLLs do not in general show increased NTD. Indeed the highest NTD seem to be associated with LBL/ IBL measurements and lower bounds. A plausible interpretation is that the $\mathrm{LBL} / \mathrm{IBL}$ are more luminous and hence visible to higher $z$, where we are less likely to detect a host galaxy.

\section{CONCLUSIONS}

We have dramatically increased the redshift completeness of this largest ever $\gamma$-ray-selected BLL sample; $\sim 44 \%$ of these BLLs now have spectroscopic redshifts and $\sim 95 \%$ have at least a strong lower bound on the redshift. These $z$ constraints show that the subset with actual spectroscopic redshifts is strongly biased to low $z$. Although we find that the measured redshifts for low-peaked BLL (LBL+IBL) do extend higher than those for the HBL with the highest synchrotron peak, our set of lower limits for both subclasses extend to yet higher $z$ than the spectroscopically solved objects. Thus, the actual redshifts for all BLLs are biased low compared to a flux-limited parent population of $\gamma$-ray BLL. This must be taken into account in any study of BLL evolution over cosmic time (M. Ajello et al., in preparation).

Many of our redshift limits rely on the common assumption that BLL hosts are standard candles. Our effort to recalibrate the standard luminosity for the Fermi BLL has resulted in fainter absolute magnitudes and, hence, more conservative minimum $z$. This implies that the standard candle assumption deserves further study, which could be best prosecuted by obtaining more high spatial resolution images of BLL with known redshifts. Our study provides a large increase in the spectroscopic redshifts, a useful precursor to such work. We find limited evidence that HBL have more luminous hosts than LBL/IBL. Whether this is intrinsic or a selection effect in the presence of a brighter, harder continuum is not yet clear.

Of course true spectroscopic redshifts are always preferable for uniform population studies. However, we suspect that much higher completeness will be difficult to attain, and will likely require novel observational techniques, as significantly more time on $8 \mathrm{~m}$ class telescopes is both expensive and likely to provide only marginally better results. In the interim, the best hope for progress lies with careful correction for selection effects in the present sample. Because of the tight correlation of synchrotron peak frequency with LAT-measured spectral index and because of the $\gamma$-ray index dependence of the LAT sensitivity, study of the relative population and evolution of different BLL subclasses will require correction for $\gamma$-ray selection effects as well as possible biases in the redshift determinations themselves. While we do not attempt such study here, our improved completeness will help in understanding these selection effects.

We find that BLL black hole mass estimates (at a given redshift) are larger than those for optically selected quasars or Fermi FSRQs. Associated with the apparent host luminosity differences, the present detections suggests that HBL host the largest mass black holes. We cannot at present tell whether these trends are a true difference in the black hole populations or luminosity-driven selection effects.

BLLs are, by definition, non-thermally dominated (NTD > 1). We find the BLL population to have significant higher NTD than the Fermi FSRQs. This claim is conservative, as the objects without redshift are likely to be at even higher NTD. Since BLLs have a range of $\gamma$-ray spectral index harder than those of FSRQs, it is perhaps surprising that this trend does not hold within the BLL class: NTD is not, on average, higher for the hardest spectrum BLL. It seems likely that, in this respect at least, BLLs are a distinct population from FSRQs, and not just the hardest spectrum objects on a continuum. In this case we might expect NTD to be controlled by the precise accident of the jet Doppler boosting. This accords well with the idea that NTD can vary for an individual source as the jet angle or effective Doppler factor vary, with relatively little change in the $\mathrm{GeV}$ spectrum. Multi-epoch, multi-wavelength studies of the brighter BLL with a range of $\gamma$-ray hardness are needed to test these ideas.

We thank the referee for a careful reading and comments that helped us add materially to the paper.

The Hobby-Eberly Telescope (HET) is a joint project of the University of Texas at Austin, the Pennsylvania State University, Stanford University, Ludwig-Maximilians-Universitaet Muenchen, and Georg-August-Universitaet Goettingen. The HET is named in honor of its principal benefactors, William P. Hobby and Robert E. Eberly. The Marcario Low Resolution Spectrograph is named for Mike Marcario of High Lonesome Optics, who fabricated several optics for the instrument but died before its completion. The LRS is a joint project of the Hobby-Eberly Telescope partnership and the Instituto de Astronoma de la Universidad Nacional Autonoma de Mexico.

Some of the data presented herein were obtained at the W. M. Keck Observatory, which is operated as a scientific partnership among the California Institute of Technology, the University of California and the National Aeronautics and Space Administration. The Observatory was made possible by the generous financial support of the W. M. Keck Foundation. The authors recognize and acknowledge the very significant cultural role and reverence that the summit of Mauna Kea has always had within the indigenous Hawaiian community. We are most fortunate to have the opportunity to conduct observations from this mountain.

This work also employs observations obtained at the Southern Astrophysical Research (SOAR) telescope, which is a joint project of the Ministério da Ciência, Tecnologia, e Inovação (MCTI) da República Federativa do Brasil, the U.S. National Optical Astronomy Observatory (NOAO), the University of North Carolina at Chapel Hill (UNC), and Michigan State University (MSU). Additional observations were made with ESO Telescopes at the La Silla Paranal Observatory under programme 077.B-0056 078.B-0275 079.B-0831 083.B-0460 084.B-0711 087.A-0573. G.C. acknowledges support from STFC grant ST/H002456/1.

We acknowledge support from NASA grants NNX09AW30G, NXX10AU09G, and NAS5-00147. A.C.S.R. is also supported under grant AST-0808050.

Facilities: Fermi, Hale (DBSP), HET, Keck:I (LRIS), NTT, VLT:Antu (FORS2).

\section{REFERENCES}

Ackermann, M., Ajello, M., Allafort, A., et al. 2011, ApJ, 743, 171 Ajello, M., Shaw, M. S., Romani, R. W., et al. 2012, ApJ, 751, 108 Appenzeller, I., Fricke, K., Fürtig, W., et al. 1998, Msngr, 94, 1 Atwood, W. B., Abdo, A. A., Ackermann, M., et al. 2009, ApJ, 697, 1071 Bajtlik, S., Duncan, R. C., \& Ostriker, J. P. 1988, ApJ, 327, 570 Beckmann, V., Engels, D., Bade, N., \& Wucknitz, O. 2003, A\&A, 401, 927 
Bohlin, R. C. 2007, in ASP Conf. Ser. 364, The Future of Photometric, Spectrophotometric and Polarimetric Standardization, ed. C. Sterken (San Francisco, CA: ASP), 315

Buzzoni, B., Delabre, B., Dekker, H., et al. 1984, Msngr, 38, 9

Dekker, H., Delabre, B., \& Dodorico, S. 1986, Proc. SPIE, 627, 339

Fioc, M., \& Rocca-Volmerange, B. 1997, A\&A, 326, 950

Fossati, G., Maraschi, L., Celotti, A., Comastri, A., \& Ghisellini, G. 1998, MNRAS, 299, 433

Ghisellini, G., \& Tavecchio, F. 2008, MNRAS, 387, 1669

Giommi, P., Padovani, P., Polenta, G., et al. 2012, MNRAS, 420, 2899

Gültekin, K., Richstone, D. O., Gebhardt, K., et al. 2009, ApJ, 698, 198

Healey, S. E., Romani, R. W., Cotter, G., et al. 2008, ApJS, 175, 97

Kaiser, J., \& Schafer, R. 1980, ITASS, 28, 105

Krisciunas, K., Sinton, W., Tholen, K., et al. 1987, PASP, 99, 887

Kurtz, M. J., \& Mink, D. J. 1998, PASP, 110, 934

Lauer, T. R., Faber, S. M., Gebhardt, K., et al. 2005, AJ, 129, 2138

León-Tavares, J., Valtaoja, E., Chavushyan, V. H., et al. 2011, MNRAS, 411, 1127

Marcha, M. J. M., Browne, I. W. A., Impey, C. D., \& Smith, P. S. 1996, MNRAS, 281,425

Meisner, A. M., \& Romani, R. W. 2010, ApJ, 712, 14

Michalitsianos, A. G., Kafatos, M., Fahey, R. P., et al. 1988, ApJ, 331, 477

Nestor, D. B., Turnshek, D. A., \& Rao, S. M. 2005, ApJ, 628, 637

Nolan, P. L., Abdo, A. A., Ackermann, M., et al. 2012, ApJS, 199, 31
O’Dowd, M., \& Urry, C. M. 2005, ApJ, 627, 97

Oke, J. B. 1990, AJ, 99, 1621

Penton, S. V., Stocke, J. T., \& Shull, J. M. 2004, ApJS, 152, 29

Plotkin, R. M., Anderson, S. F., Brandt, W. N., et al. 2010, AJ, 139, 390

Rector, T. A., Stocke, J. T., Perlman, E. S., Morris, S. L., \& Gioia, I. M. 2000, AJ, 120, 1626

Sbarufatti, B., Treves, A., \& Falomo, R. 2005a, ApJ, 635, 173

Sbarufatti, B., Treves, A., Falomo, R., et al. 2005b, AJ, 129, 559

Schlafly, E. F., \& Finkbeiner, D. P. 2011, ApJ, 737, 103

Schlegel, D. J., Finkbeiner, D. P., \& Davis, M. 1998, ApJ, 500, 525

Shaw, M. S., Romani, R. W., Cotter, G., et al. 2012, ApJ, 748, 49 (S12)

Shaw, M. S., Romani, R. W., Healey, S. E., et al. 2009, ApJ, 704, 477

Shen, Y., Richards, G. T., Strauss, M. A., et al. 2011, ApJS, 194, 45

Stickel, M., Fried, J. W., \& Kuehr, H. 1993, A\&AS, 98, 393

Szokoly, G. P., Bergeron, J., Hasinger, G., et al. 2004, ApJS, 155, 271

Tody, D. 1986, Proc. SPIE, 627, 733

Urry, C. M., \& Padovani, P. 1995, PASP, 107, 803

Urry, C. M., Scarpa, R., O’Dowd, M., et al. 2000, ApJ, 532, 816

Valdes, F. 1986, Proc. SPIE, 627, 749

Valdes, F. 1992, in ASP Conf. Ser. 25, Astronomical Data Analysis Software and Systems I, ed. D. M. Worrall, C. Biemesderfer, \& J. Barnes (San Francisco, CA: ASP), 417

Weymann, R. J., Jannuzi, B. T., Lu, L., et al. 1998, ApJ, 506, 1

Yuan, H. B., \& Liu, X. W. 2012, MNRAS, 425, 1763 